\title{
ÉVALUATION ÉCONOMIQUE DES PLANS DE GESTION PISCICOLE.
}

\author{
C. ARMAND (1), F. BONNIEUX (1), T. CHANGEUX (2)
}

\begin{abstract}
(1) INRA-Economie, rue Adolphe Bobierre, CS 61103 - 35011 RENNES Cedex, France.
(2) Conseil Supérieur de la Pêche, Service Technique, 134 avenue de Malakoff 75116 PARIS, France.
\end{abstract}

Reçu le 10 juillet 2000

Accepté le 29 janvier 2001
Received 10 July, 2000

Accepted 29 January, 2001

\section{RÉSUMÉ}

En France, la gestion de la pêche en eau douce est généralement confiée aux associations de pêcheurs qui agissent sous le contrôle de l'Etat. Le Conseil Supérieur de la Pêche a développé une méthode synthétique d'élaboration de plans de gestion piscicole qui favorisent la reproduction naturelle et le maintien des populations sauvages. Ces plans s'appuient sur des opérations de protection des habitats et de restauration du milieu dont il convient d'évaluer la rentabilité sociale. Ce qui conduit à estimer la valeur du poisson sauvage (c'est à dire né dans le milieu naturel, de parents eux-mêmes sauvages). Cette valeur intègre deux composantes principales, une valeur d'usage qui dérive de la pratique de la pêche et une valeur de non-usage qui découle de son existence même et qui intéresse une population plus vaste que celle des seuls pêcheurs.

Les bénéfices potentiels engendrés par la présence de poissons sauvages sont estimés par la méthode d'évaluation contingente. Les résultats empiriques sont basés sur un échantillon de 1629 pêcheurs qui ont participé à une enquête postale. Le Consentement A Payer (CAP) pour le poisson sauvage est une fonction croissante du revenu, de la valeur du matériel et de la distance parcourue pour pêcher. De plus on note que les amateurs de brochets et de truites (espèces repères de l'étude) ainsi que ceux qui pêcheraient plus souvent s'il y avait du poisson sauvage ont, toutes choses égales, par ailleurs un $C A P$ plus élevé. La valeur moyenne du CAP varie selon le modèle retenu de $50 \mathrm{~F}$ à $100 \mathrm{~F}$ par pêcheur et par an. Ce CAP correspond à un concept de valeur totale qui inclut une composante de valeur d'usage et de valeur de non-usage. Certains pêcheurs sont prêts à payer sans pour autant marquer une préférence pour la pêche au poisson sauvage. Leur CAP est associé à une valeur de non-usage et correspond à la valeur d'existence du poisson sauvage. Celle-ci est comprise entre $20 \mathrm{~F}$ et $50 \mathrm{~F}$ par pêcheur et par an. Un calcul simple montre que les bénéfices des pêcheurs sont du même ordre que les coûts de fonctionnement associés aux plans de gestion piscicole.

Mots-clés : plan de gestion piscicole, évaluation contingente, valeur d'usage, valeur d'existence, rentabilité sociale, poisson sauvage, brochet, truite. 


\title{
AN ECONOMIC ASSESSMENT OF INLAND FISHERIES MANAGEMENT SCHEMES.
}

\begin{abstract}
In France, angling societies are, under the supervision of the government, generally in charge of inland fisheries management. The National Council of Fishing has developed a series of guidelines to design management schemes which favour natural reproduction processes and the preservation of wild fish stocks. They rely on habitat protection and ecosystem restoration programmes involving public expenditures whose profitability has to be assessed. This leads to estimate the value of wild fish (born in the natural environment from parents wild fish themselves). This value integrates two main components, an use value which is derived from angling and a non use value which stems from the existence of wild fish and concerns a broader population than the anglers one.
\end{abstract}

Potential benefits stemming from the existence of wild fish populations are estimated using the contingent valuation method. Empirical results are based on a sample of 1629 anglers who participated in a mail survey. Willingness-To-Pay (WTP) for wild fish increases with income, cost of equipment and trip distance. Other things being equal, those preferring fish such as pike and trout (reference species of the study), and those preferring to angle wild fish are likely to pay an additional amount. Mean WTP ranges from FF 50 to FF 100 per year, depending on model specification. This money amount is associated with the concept of total value which includes a use and a non use component. Some anglers are willing to pay but are not interested in fishing wild fish. For these people, WTP states a non use value and is associated with the existence of wild fish. The corresponding amount is estimated to be between FF 20 and FF 50. A straightforward calculation shows that anglers' potential benefits are of the same magnitude than the operating costs associated to management schemes.

Key-words : fishing management scheme, contingent valuation, use value, existence value, social profitability, wild fish, pike, trout.

\section{INTRODUCTION}

Dans les eaux douces, le droit de pêche est attribué en France, à l'Etat dans un nombre limité de cas (Article L. 235-1 ${ }^{1}$ ) et en règle générale aux propriétaires riverains pour les cours d'eau et les canaux ou aux propriétaires du fonds pour les plans d'eau (Article L. 235-4'). L'exercice de ce droit implique une obligation de gestion des ressources piscicoles qui comporte l'établissement d'un plan de gestion (Article L. 233-3') qui s'inscrit dans un schéma départemental de vocation piscicole, qui lui même doit être cohérent avec les orientations du bassin (Articles L. 233-1 et 233-2'). Concernant la pêche récréative, les détenteurs du droit de pêche le cèdent généralement à l'une des 4200 associations agréées (AAPPMA ${ }^{2}$ ) regroupées en quatre-vingt treize fédérations départementales qui coordonnent les actions des associations. Toutes ces actions visent non seulement à l'organisation de la pêche et à son développement, mais aussi à la protection du patrimoine piscicole et à la valorisation des milieux aquatiques (Article L. 234-4 ${ }^{1}$ ). Dans cette organisation relativement complexe, le Conseil Supérieur de la Pêche joue un rôle d'expert en apportant un soutien technique aux fédérations et aux associations.

La politique de gestion a longtemps privilégié le déversement de poissons pour faire face à la demande de pêche, ce qui a permis, malgré la dégradation des milieux, de

\footnotetext{
${ }^{1}$ Loi n 84-512 du 29 juin 1984 dite « loi pêche » (voir livre 2 du code rural).

${ }^{2}$ Association Agréée pour la Pêche et la Protection du Milieu Aquatique.
} 
maintenir un certain niveau d'offre de pêche. Cette politique palliait partiellement l'effet des dommages en satisfaisant la demande de certains pêcheurs sans se soucier de la qualité des milieux aquatiques. Cette forme de gestion utilise le milieu naturel comme un simple support d'accueil temporaire pour un stock de poissons dont la seule fonction est d'être pêché. La remise en cause de cette politique de gestion a conduit à partir de 1994 à une réorientation qui passe désormais par la mise en place de plans de gestion piscicole favorisant la reproduction naturelle et le maintien des populations sauvages. Elle marque donc le passage d'une gestion de la ressource privilégiant la composante quantitative de l'offre de pêche à une gestion du milieu centrée sur sa dimension qualitative. Sa mise en œuvre passe par la restauration des habitats et l'amélioration de la biodiversité, ce qui implique des coûts de protection et de restauration qui relèvent de dépenses d'investissement et d'entretien. L'évaluation économique de ces plans conduit à s'interroger sur la nature et la valeur des bénéfices qu'ils sont susceptibles d'engendrer. Ce qui conduit à s'interroger sur la valeur des poissons d'origine sauvage, c'est à dire nés dans le milieu naturel, de parents eux-mêmes sauvages.

Après avoir présenté le programme des plans de gestion qui se met en place, on proposera à partir du cas des départements de l'Hérault et de l'Indre, une estimation de la valeur du poisson sauvage. Comme les plans se mettent en place et n'ont donc pas produit tous les effets escomptés, on se situe dans une perspective ex ante, cette estimation peut s'appuyer sur une évaluation contingente. Enfin, les coûts de ces plans seront comparés aux bénéfices des pêcheurs de ces deux départements ce qui permet de dégager des conclusions provisoires sur leur rentabilité sociale.

\section{PROGRAMME NATIONAL DES PLANS DE GESTION}

HOLL et AUXIETRE (non daté) ont développé une méthode de diagnostic pour coordonner la mise en place des plans de gestion locaux au travers d'un document de programmation départemental, qui contient les bases techniques nécessaires pour que chaque détenteur de droit de pêche puisse élaborer son plan de gestion, le faire approuver par l'administration, et le mettre en œuvre avec l'appui de nouveaux partenaires financiers. II comporte deux volets (Figure 1) : le Plan Départemental pour la Protection du milieu aquatique et la Gestion des ressources piscicoles (PDPG) et le Plan Départemental pour la Promotion du Loisir-pêche (PDPL).

Depuis 1994, plus de la moitié des départements s'est engagée dans ce programme avec l'aide technique et financière du CSP. Le PDPG fait un bilan des potentialités du milieu, des déficits actuels, et des travaux de réhabilitation à réaliser pour les combler. Le PDPL traite ces potentialités en offre de pêche et les confronte avec les possibilités de développement du loisir en vue d'enrayer la diminution du nombre de pêcheurs (JANTZEN, 1998). Ces documents sont rédigés par un ingénieur, conseiller technique engagé par la fédération et chargé de mettre en œuvre la méthode du CSP. Ces documents sont régulièrement réévalués grâce à différents suivis volontaires de captures ou d'enquêtes menées auprès des pêcheurs.

\section{PDPG}

Dans le PDPG, le conseiller technique scinde le réseau en unités homogènes, appelées « contextes piscicoles ». II fait son diagnostic à partir d'une espèce repère, indicatrice de la situation globale du milieu (truite en contexte salmonicole, brochet en contexte cyprinicole). Le diagnostic consiste, pour chaque contexte, à comparer l'Effectif Potentiel de Poissons Adultes (EPPA) au nombre actuel. II utilise pour ce faire les résultats contenus dans le Schéma Départemental de Vocation Piscicole (SOUCHON et TROCHERIE, 1990). 


\section{I- Plan départemental pour la protection du milieu aquatique et la gestion des ressources piscicoles (PDPG)}

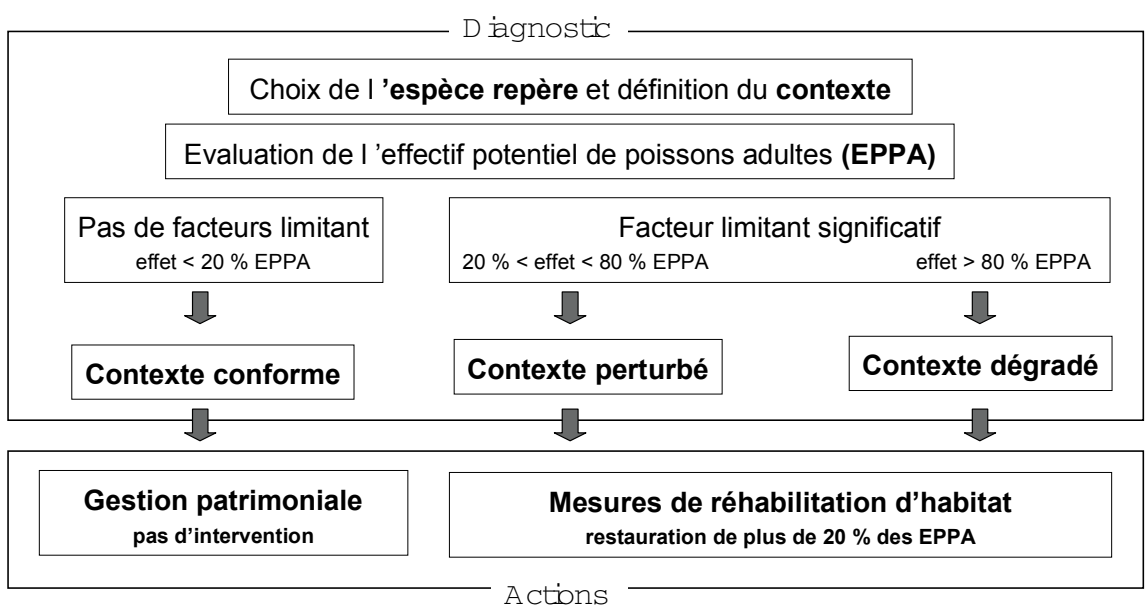

\section{II- Plan départemental pour la promotion du loisir-pêche} (PDPL)

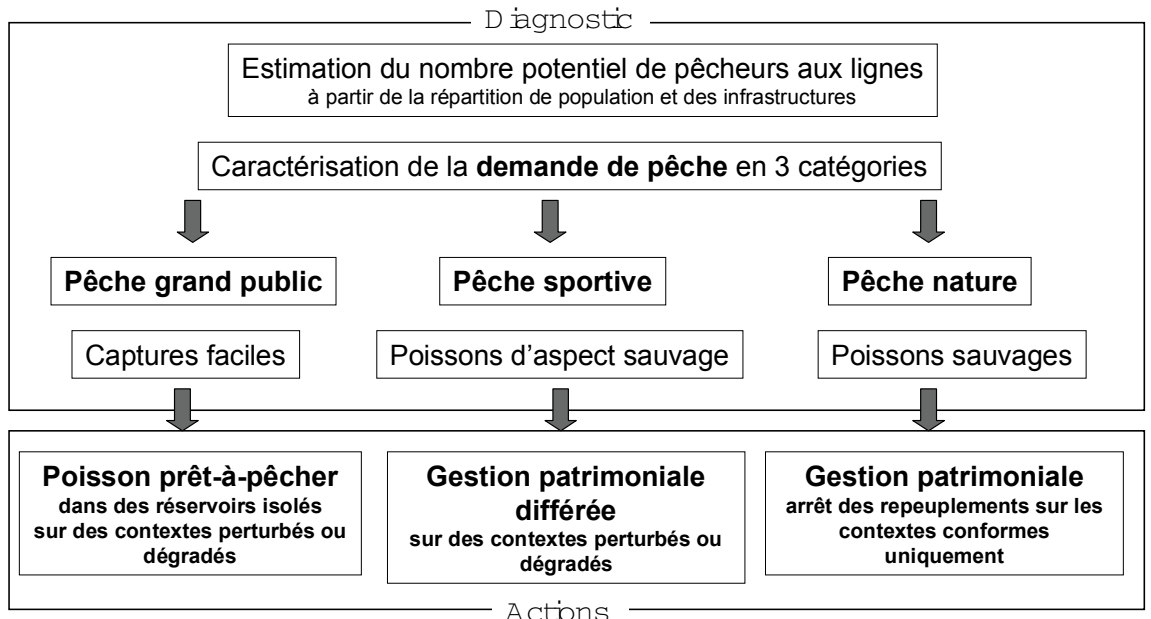

\section{Figure 1}

Etapes de réalisation des plans de gestion.

\section{Figure 1}

Steps for the design of management schemes.

Lorsqu'aucun facteur limitant n'est sensible (effet inférieur à $20 \%$ de l'EPPA), le contexte est considéré comme étant conforme et le conseiller technique propose aux gestionnaires de conduire une gestion patrimoniale en cessant toute intervention. La gestion patrimoniale implique l'arrêt des repeuplements qui sont inutiles voire préjudiciables aux poissons sauvages dans cette situation. C'est la meilleure garantie du maintien du contexte en situation conforme. Cela suppose néanmoins que les prélèvements sont en relation avec la capacité du milieu, celui-ci étant protégé des agressions qui pourraient survenir. 
Dans les situations les moins favorables, le contexte est considéré comme perturbé (effet limitant de $20 \%$ à $80 \%$ de l'EPPA ) ou dégradé (effet limitant supérieur à $80 \%$ de I'EPPA). Le conseiller technique détaille alors les travaux de réhabilitation nécessaires pour rétablir sensiblement le niveau de poissons adultes du contexte (gain supérieur à $20 \%$ de I'EPPA). Chaque intervention (aménagement de frayères, construction de passes à poissons, etc.) est évaluée financièrement, pour être proposée aux Agences de l'Eau, aux Régions, à l'Etat et aux autres partenaires financiers intéressés par la réhabilitation de la qualité des milieux aquatiques.

Une fois le document technique produit, la fédération arrête ses orientations politiques pour une période de cinq ans dans son plan des actions nécessaires. Elle y inscrit les contextes à gestion patrimoniale, où les repeuplements seront stoppés, et les contextes où des travaux doivent être entrepris, en détaillant pour chaque intervention le coût et le gain attendus en effectif de poissons adultes supplémentaires.

\section{PDPL}

Dans le PDPL, le conseiller technique estime le nombre de pêcheurs potentiels à partir de la répartition de la population et des grands axes de déplacements. En s'appuyant sur les derniers résultats de segmentations de la population des pêcheurs (ANONYME, 1996), la méthode retient trois types de pêcheurs distincts : le pêcheur grand public, intéressé par le poisson prêt à pêcher, dans un périmètre peu éloigné de son domicile, le pêcheur sportif, soucieux d'avoir un poisson d'aspect sauvage à une heure maximum de déplacement en voiture et enfin le pêcheur nature, attaché à la qualité du site de pêche et des prises, qui est prêt à se déplacer sur de grandes distances ${ }^{3}$.

La répartition spatiale de ces pêcheurs détermine des zones de services dans lesquelles on croise le nombre de poissons nécessaires pour satisfaire la demande et le nombre de poissons adultes produits par les contextes. Pour chaque zone de service, des propositions sont faites en termes de nombre de pêcheurs à former, nombre de structures d'accueil nécessaire, linéaire de parcours à ouvrir, etc., pour satisfaire la demande. Ces propositions sont adaptées aux potentialités du milieu et ne doivent pas modifier les choix de gestion faits dans le PDPG. On propose de préférence aux pêcheurs nature des contextes à gestion patrimoniale, aux pêcheurs sportifs des contextes perturbés ou dégradés, à gestion patrimoniale différée, où les repeuplements sont admis, enfin aux pêcheurs grand public une pêche réservoir avec du poisson prêt à pêcher. Comme précédemment, la fédération arrête ses orientations politiques pour une période de cinq ans dans son plan des actions de type loisir.

\section{VALEUR DU POISSON SAUVAGE}

L'intégration du poisson sauvage dans le calcul économique soulève un certain nombre de difficultés puisqu'il s'agit d'un bien non-marchand. La valeur du poisson sauvage a deux composantes principales. La première est liée à la pratique de la pêche et donc intégrée à une valeur d'usage tandis que la seconde relève de son existence même et n'intéresse pas que les seuls pêcheurs mais une population plus vaste. En effet, indépendamment de tout usage présent ou futur, des individus peuvent accorder une valeur à la biodiversité et au poisson sauvage. Ce concept de valeur de non-usage inclut différentes notions qui ne sont pas toujours clairement identifiées: valeur d'existence, valeur intrinsèque, ou valeur de préservation (FREEMAN, 1993).

\footnotetext{
${ }^{3}$ Cette typologie des pêcheurs définie par RICHARD (non daté) est utilisée dans les plans de gestion.
} 
L'offre effective de poissons sauvages va dépendre de la mise en œuvre effective de la politique de restauration du milieu et de son succès. Cette incertitude sur l'offre d'actif naturel se combine à une incertitude touchant la demande. Les pêcheurs n'ont pas en effet une pleine connaissance de leurs préférences futures et des stocks de poissons sauvages qui seront disponibles. Dans l'avenir, continueront-ils à pêcher ? Et si oui, plus ou moins souvent que maintenant ? Dans ce contexte, pour mener à bien l'évaluation ex ante d'une politique de restauration, il convient de distinguer trois catégories de pêcheurs (BONNIEUX et DESAIGUES, 1998) :

- les usagers certains qui accordent à l'actif naturel une valeur supérieure à la seule valeur d'usage car ils désirent que ce bien soit préservé,

- les usagers incertains mais qui veulent conserver une option future et donc la possibilité d'être usagers,

- les individus qui sont sûrs de ne jamais faire usage de l'actif naturel mais qui, pour des raisons diverses (altruisme, éthique), lui attribuent une valeur de conservation.

Dans cette recherche, on s'est attaché à la population des seuls pêcheurs qui, pour une large majorité, se classent dans la catégorie des usagers certains. Deux départements, l'Hérault et l'Indre, correspondant à des situations géographiques et halieutiques contrastées, ont été sélectionnés. Le premier entretient une demande de pêche orientée en faveur de la truite alors que les conditions naturelles sont peu favorables à cette espèce. Dans le second, la demande est plus diversifiée et les contextes sont adaptés au brochet.

\section{Méthode et protocole}

La méthode d'évaluation contingente a été retenue pour évaluer le consentement à payer $(C A P)$ des pêcheurs. Une enquête sur site et par téléphone auprès d'un nombre restreint de pêcheurs (treize au total) a permis de définir le bien à valoriser et de décrire le scénario. Elle a abouti à la mise au point d'un questionnaire avec une question ouverte pour révéler le $C A P$, qui a été administré par voie postale. Ce protocole s'écarte donc sur plusieurs points des recommandations de la National Oceanic and Atmospheric Administration (ARROW et al., 1993), qui n'intègrent pas il est vrai un certain nombre d'acquis méthodologiques récents (BATEMAN et WILLIS, 1999). L'information disponible au préalable, a permis de s'affranchir de l'utilisation de groupes cibles dans la première phase. Cette technique s'avère d'ailleurs peu efficace du fait de l'émergence de leaders dans les petits groupes d'individus. Lorsque le bien à valoriser est familier aux personnes interrogées et qu'un mécanisme de marché existe déjà, la question ouverte est un mécanisme de révélation appropriée. C'est pour ces raisons qu'elle est employée fréquemment pour la pêche de loisir (WILLIS et GARROD, 1999).

Le questionnaire est organisé selon un plan en quatre sections, classique en analyse contingente. La première section vise à recueillir les données socio-économiques, à l'exception de celles considérées comme confidentielles, qui permettent de décrire la personne interrogée. La seconde vise à préciser son activité de pêche en s'intéressant en particulier à l'espèce qui est la plus recherchée. La troisième section est réservée au marché contingent. Après avoir défini le poisson sauvage, le scénario hypothétique est présenté au pêcheur qui est interrogé sur le montant de son CAP. La dernière section est réservée à des données plus confidentielles (revenu, niveau de formation) qui ne sont pas abordées en début de questionnaire car cela peut entraîner des réactions de rejet.

Le scénario hypothétique est la partie la plus importante du questionnaire. II doit décrire de manière précise la structure du marché contingent et la nature du bien à valoriser. II doit aussi renseigner la personne interrogée sur le mode de paiement retenu 
pour exprimer le $C A P$. En effet, la méthode d'évaluation contingente est aussi un exercice de communication puisqu'il s'agit de faire révéler aux personnes interrogées leurs préférences pour le changement hypothétique proposé. Dans le cas de la pêche de loisir, la situation vécue est connue par l'ensemble des pêcheurs, ce qui facilite la construction du scénario contingent. En effet, les pêcheurs sont familiarisés avec la ressource et la valeur que l'on cherche à mesurer est dérivée d'un usage direct du milieu naturel. Une fois levées les ambiguïtés, la définition du poisson sauvage ne pose pas de problème particulier et se révèle moins délicate que dans de nombreuses études qui s'intéressent à une population moins ciblée que celle des pêcheurs. Le poisson sauvage est défini dans le questionnaire par comparaison au poisson de repeuplement, dans les termes suivants : " le poisson sauvage est né et a grandi dans la rivière de parents eux-mêmes nés dans la rivière. Le poisson de repeuplement n'est pas un poisson sauvage ". II est aussi indispensable de définir la situation de référence en précisant l'état actuel de la qualité des poissons dans les rivières et l'amélioration dont on propose la valorisation. La politique menée jusqu'à présent dans le domaine halieutique a privilégié le repeuplement. Au contraire, dans ce questionnaire, on demande aux pêcheurs combien ils sont prêts à payer pour « restaurer le milieu aquatique (de manière à ce que) les poissons sauvages puissent vivre et se reproduire dans le milieu naturel ». Le choix d'un véhicule de paiement réaliste et neutre ne soulève pas de difficulté particulière puisque les pêcheurs ont l'habitude de payer pour bénéficier de la ressource. Une augmentation du prix de la carte de pêche est donc le véhicule de paiement le plus adapté pour valoriser une amélioration de la qualité des poissons. Après avoir défini le scénario et le mode de paiement de manière claire et précise, on a posé la question suivante : « combien seriez-vous prêt à payer en plus du prix de la carte de pêche pour pêcher du poisson sauvage ?».

Le choix d'une enquête par voie postale pour valoriser le poisson sauvage constitue un compromis. Cette technique largement utilisée dans ce type d'études est moins coûteuse que l'interview en face-à-face et permet de présenter un matériel plus complexe que dans une enquête par téléphone (POLLOCK et al., 1994). La personne interrogée dispose aussi de plus de temps pour construire sa réponse si elle reçoit le questionnaire par voie postale. Cet avantage peut néanmoins se transformer en inconvénient, car cela lui laisse aussi plus de temps pour adopter un comportement stratégique. Elle évite le biais de l'enquêteur mais le taux de réponse est généralement faible d'où un biais d'autosélection. En effet, on constate en général que le taux de réponse diminue avec l'âge et augmente avec le revenu et le niveau formation. Afin de réduire le taux de non-réponse et le nombre de réponses incohérentes, on a introduit un système de gratification. Pour motiver les pêcheurs, on a institué une loterie dotée d'un prix de $5000 \mathrm{~F}$ en matériel de pêche.

L'échantillonnage a été réalisé à partir des fichiers d'adresses des pêcheurs qui fournissent aussi leur âge. Un échantillon aléatoire simple de 5000 pêcheurs a été constitué pour chaque département, le taux de sondage est de $36 \%$ dans l'Indre et de $32 \%$ dans l'Hérault. Ce tirage a permis de constituer des échantillons représentatifs des populations de pêcheurs pour le critère âge, le seul qui puisse être contrôlé. Un total de 1629 questionnaires ont été renvoyés par les pêcheurs, ce qui correspond à un taux de réponse de $17 \%$ pour l'ensemble des deux départements. Pour le critère âge, l'échantillon des questionnaires renvoyés est caractérisé par une sur-représentation des individus âgés de 20 à 40 ans et corrélativement une sous-représentation des personnes âgées de plus de 50 ans. Le biais d'échantillonnage s'accentue avec l'âge.

\section{Comportement des pêcheurs et préférence pour le poisson sauvage}

On observe des différences importantes de comportement selon le lieu de résidence des pêcheurs, en analysant en particulier l'effort de pêche et la distance entre le domicile et le site le plus fréquenté. L'effort de pêche est mesuré par le nombre annuel de sorties (46 pour l'ensemble de l'échantillon avec une médiane de 30 sorties) et la durée moyenne 
d'une sortie (5 heures). Les résidents de l'Indre sont des pêcheurs de proximité, qui sortent plus souvent (48 sorties) et près de chez eux $(18 \mathrm{~km})$. Les pêcheurs n'habitant ni l'Indre ni l'Hérault sont comme on pouvait s'y attendre des pêcheurs moins assidus (41 sorties), mais qui parcourent une distance plus élevée $(133 \mathrm{~km})$. Les pêcheurs de l'Hérault sont dans une situation intermédiaire en termes de sorties (46 sorties) et de distance moyenne parcourue $(53 \mathrm{~km})$. Les coûts de transport constituent le poste de dépenses le plus important et sont donc beaucoup plus élevés pour les non-résidents que pour les autres catégories. Comme prévu, les individus qui pêchent loin de leur lieu de résidence disposent d'un revenu plus élevé que les autres. Corrélativement, la valeur de leur matériel est supérieure à celle des autres pêcheurs, $6819 \mathrm{~F}$ et $4925 \mathrm{~F}$ respectivement.

La description des comportements peut être affinée en considérant les captures et le nombre de poissons gardés. II convient ici de stratifier l'échantillon selon l'espèce recherchée. La truite est privilégiée par $40 \%$ des pêcheurs (55\% dans l'Hérault), le brochet recherché par $20 \%$ (24\% dans l'Indre), les $40 \%$ restants ciblant d'autres espèces, cyprinidés en particulier. Ceci corrobore des résultats antérieurs (GAMOND, 1991). Les truites sont beaucoup plus abondantes que les brochets, ce qui explique en partie les différences dans le nombre de captures. Les pêcheurs de truites déclarent capturer en moyenne 67 poissons par an, 28 étant conservés. Les pêcheurs de brochet ne déclarent de leur côté que 15 captures, 5 poissons étant conservés. II faut toutefois noter que cette catégorie de pêcheurs vise d'autres carnassiers tels que le sandre, la perche, le chevaine ou le black bass.

La préférence pour le poisson sauvage est très marquée et concerne $77 \%$ de l'échantillon. Elle est sensiblement plus élevée chez les résidents de l'Hérault où les problèmes de pollution de l'eau sont plus importants. Peu d'individus déclarent ne pas préférer pêcher du poisson sauvage (moins de $2 \%$ ), les autres ne se prononcent pas (« ça m'est égal », $16 \%$, « ne sait pas », $3 \%$ ). Les raisons qui motivent la préférence pour le poisson sauvage peuvent être facilement classées. La première catégorie de raisons évoquées par les pêcheurs concernent la combativité, la vigueur et la résistance du poisson sauvage qui le rendent difficile à attraper ( $42 \%$ des premières réponses). La deuxième raison donnée est liée à la pêche elle-même : le poisson sauvage rend la pêche plus intéressante, plus sportive et technique $(11 \%)$. La troisième fait référence à la caractéristique "sauvage " du poisson $(10 \%)$, les pêcheurs trouvent le poisson plus naturel et adapté à son milieu. Certains pêcheurs ont avancé plusieurs raisons pour expliquer l'attrait du poisson sauvage. Dans cette série d'éléments complémentaires, une place importante est donnée à la qualité gustative puisque plus de $20 \%$ des pêcheurs qui donnent une deuxième raison, la citent. La présence de poissons sauvages est susceptible d'augmenter la demande de pêche puisque $57 \%$ des pêcheurs préférant le poisson sauvage affirment qu'ils iraient pêcher plus souvent $(40 \%$ dans l'Indre et $60 \%$ dans l'Hérault).

Parmi les 27 individus qui affirment ne pas préférer le poisson sauvage, 13 seulement expliquent leur attitude par le fait qu'ils préfèrent la quantité à la qualité ou bien qu'ils ne sont pas capables de voir la différence entre poisson sauvage et poisson de repeuplement. A quelques exceptions près, les individus indifférents au poisson sauvage n'expliquent pas leur choix.

\section{Estimation du CAP}

Pour l'ensemble de l'échantillon (1 629 individus), on dénombre 271 soit $17 \%$ de valeurs manquantes, 408 valeurs nulles et 396 valeurs inférieures à $50 \mathrm{~F}$. La distribution du CAP est asymétrique avec une médiane $(50 \mathrm{~F})$ inférieure à la moyenne $(100 \mathrm{~F})$ et une accumulation de réponses autour des valeurs les plus faibles. Ces valeurs et en particulier la médiane sont robustes lorsque l'on tronque l'échantillon en éliminant les individus les plus jeunes. Le CAP moyen varie sensiblement selon l'espèce recherchée (Tableau I). II 
est plus élevé pour le pêcheur de truites que de brochets, les pêcheurs qui s'intéressent à d'autres espèces déclarant de leur côté les valeurs les plus faibles. Le CAP par poisson conservé doit être interprété avec prudence, puisque ce rapport combine les résultats de deux estimations. Le CAP par brochet conservé est nettement plus élevé que le CAP par truite conservée. Cette différence signifie que les pêcheurs attribuent une valeur plus élevée au brochet sauvage qu'à la truite sauvage, ce qui est cohérent eu égard à la plus grande rareté du brochet.

\section{Tableau I}

Le consentement à payer (CAP) moyen des pêcheurs (en F) selon l'espèce recherchée.

\section{Table I}

Mean willingness to pay (WTP) of anglers (in FF) by type of fish.

\begin{tabular}{|c|c|c|}
\hline $\begin{array}{c}\text { Espèce } \\
\text { Type of fish }\end{array}$ & $\begin{array}{c}\text { CAPlan } \\
\text { WTP/year }\end{array}$ & $\begin{array}{c}\text { CAP/poisson gardé } \\
\text { WTP/fish kept }\end{array}$ \\
\hline $\begin{array}{c}\text { Brochet } \\
\text { Pike }\end{array}$ & 90 & 18 \\
\hline $\begin{array}{c}\text { Truite } \\
\text { Trout }\end{array}$ & 122 & 4,35 \\
\hline $\begin{array}{c}\text { Autres } \\
\text { Other }\end{array}$ & 75 & \\
\hline $\begin{array}{c}\text { Total } \\
\text { All }\end{array}$ & 99 & \\
\hline
\end{tabular}

La modélisation économétrique permet de dégager les principaux facteurs qui expliquent le montant du CAP (ARMAND et BONNIEUX, 1999). Le Tableau II fournit pour trois modèles les résultats les plus significatifs. La variable dépendante est égale au $C A P$ dans le modèle linéaire, à une transformation logarithmique du CAP dans le modèle semi$\log ^{4}$ et à une transformation dite de Box Cox du CAP dans le modèle de Box Cox ${ }^{5}$. Les variables explicatives décrivent le pêcheur (âge, revenu) et sa pratique de la pêche (préférence pour la truite ou le brochet, distance du domicile au site le plus fréquenté, valeur du matériel et augmentation de l'effort de pêche si le projet est réalisé). Ces modèles, bien que leur qualité prédictive soit limitée (faible valeur du coefficient de corrélation multiple) permettent de faire un certain nombre de remarques sur la nature des facteurs qui jouent de façon significative sur le CAP. Pour ce faire on s'appuie sur le test de Student. Le revenu a un effet positif sur le CAP mais en revanche on n'observe pas d'effet significatif de l'âge sur le $C A P$. Les personnes les plus âgées sont moins enclines à annoncer un $C A P$ positif, mais quand elles le font, ce sont les autres déterminants qui influencent sa valeur et en particulier le revenu. On a à faire à un processus en deux étapes, on décide tout d'abord d'annoncer ou non une valeur non-nulle, si oui on la fixe dans un deuxième temps. Le CAP apparaît logiquement comme une fonction croissante de l'effort de pêche comme le montre le signe des coefficients des variables valeur du matériel et distance du domicile au site le plus fréquenté, ainsi que de la préférence pour la truite ou le brochet. De plus la catégorie des pêcheurs qui envisagent d'augmenter leur effort est toutes choses égales par ailleurs caractérisée par un CAP plus élevé. Le CAP

\footnotetext{
${ }^{4}$ La variable dépendante est égale à $\log (C A P+K)$ où $K$ est une constante arbitraire, ce qui permet de traiter les $C A P$ égaux à zéro. On a pris ici $K=10 \mathrm{~F}$.

${ }^{5}$ La variable dépendante est égale à $\left(C A P^{\prime}-1\right) / /$ où $/$ est un paramètre compris entre 0 et 1 . Sa valeur est déterminée par une procédure statistique, $I=0,7$.
} 
moyen estimé dépend du modèle retenue, il varie de 45,9 F avec le modèle semilogarithmique à 107,5 F avec le modèle linéaire. Rappelons que seul ce dernier fournit une estimation sans biais du CAP moyen.

\section{Tableau II}

Estimation du consentement à payer (CAP) moyen.

Table II

Estimation of mean willingness to pay (WTP).

\begin{tabular}{|c|c|c|c|}
\hline $\begin{array}{l}\text { Modèle } \\
\text { Model }\end{array}$ & $\begin{array}{l}\text { Linéaire } \\
\text { Linear }\end{array}$ & $\begin{array}{l}\text { Log linéaire } \\
\text { Semi-log }\end{array}$ & $\begin{array}{l}\text { Box Cox } \\
\text { Box Cox }\end{array}$ \\
\hline $\begin{array}{l}\text { Constante } \\
\text { Intercept }\end{array}$ & $\begin{array}{l}-10,30 \\
(0,48)\end{array}$ & $\begin{array}{l}3.37 \\
(21,3)\end{array}$ & $\begin{array}{c}5,93 \\
(1,38)\end{array}$ \\
\hline $\begin{array}{l}\text { Age (années) } \\
\text { Age (years) }\end{array}$ & $\begin{array}{c}0,35 \\
(1,03)\end{array}$ & $\begin{array}{l}-0,003 \\
(1,21)\end{array}$ & $\begin{array}{c}0,03 \\
(0,46)\end{array}$ \\
\hline $\begin{array}{l}\text { Revenu mensuel (1 } 000 \mathrm{~F}) \\
\text { Monthly income (FF } 1000)\end{array}$ & $\begin{array}{l}8,55 \\
(2,56)\end{array}$ & $\begin{array}{l}0,056 \\
(2,28)\end{array}$ & $\begin{array}{c}1,77 \\
(2,66)\end{array}$ \\
\hline $\begin{array}{l}\text { Préférence pour la truite ou le brochet } \\
\text { Oui }=1, \text { Non }=0 \\
\text { Preference for trout or pyke } \\
\text { Yes }=1, \text { No }=0\end{array}$ & $\begin{array}{l}28,82 \\
(2,45)\end{array}$ & $\begin{array}{l}0,226 \\
(2,63)\end{array}$ & $\begin{array}{c}6,52 \\
(2,79)\end{array}$ \\
\hline $\begin{array}{l}\text { Augmentation de la fréquentation s'il y a } \\
\text { du poisson sauvage. Oui }=1 \text {, Non }=0 \\
\text { Increase in number of visits if there is } \\
\text { wild fish. Yes }=1, \text { No }=0\end{array}$ & $\begin{array}{l}47,82 \\
(3,75)\end{array}$ & $\begin{array}{l}0,487 \\
(5,21)\end{array}$ & $11,43(4,50)$ \\
\hline $\begin{array}{l}\text { Distance du domicile }(\mathrm{km}) \\
\text { Trip distance }(\mathrm{km})\end{array}$ & $\begin{array}{c}0,15 \\
(2,76)\end{array}$ & $\begin{array}{l}0,0008 \\
(1,90)\end{array}$ & $\begin{array}{l}0,027 \\
(2,52)\end{array}$ \\
\hline $\begin{array}{l}\text { Valeur du matériel } \\
\text { Value of equipment }\end{array}$ & $\begin{array}{l}0,001 \\
(2,06)\end{array}$ & $\begin{array}{l}0,000002 \\
(0,44)\end{array}$ & $\begin{array}{l}0,0002 \\
(1,66)\end{array}$ \\
\hline $\mathrm{R}^{2}(\%)$ & 5,7 & 5,8 & 6,2 \\
\hline $\begin{array}{l}\text { CAP estimé (F) } \\
\text { Estimated WTP (FF) }\end{array}$ & 107,5 & 45,9 & 80,9 \\
\hline
\end{tabular}

Note : l'échantillon utilisé compte 1286 individus. La valeur du t de Student est donnée entre parenthèses. $R^{2}$ est égal au carré du coefficient de corrélation multiple. Note: the sample size is 1 286. Student $t$ values in parentheses. $R^{2}$ is the coefficient of determination.

Une large majorité de pêcheurs marque une préférence pour le poisson sauvage et constitue la catégorie des « usagers potentiels » de cette ressource. Par ailleurs, un nombre relativement élevé de pêcheurs (184) n'ont pas de préférence pour la pêche au poisson sauvage, et n'iraient pas pêcher plus souvent. Certains (109) annoncent cependant un CAP strictement positif. II existe donc une autre source de variation du bienêtre qui n'est pas directement liée à l'augmentation du nombre de sorties de pêche, ni à la pêche de poissons sauvages en tant qu'activité récréative. Leur annonce de $C A P$ correspond donc à une variation d'utilité, indépendante de l'usage de la ressource. 
On définit donc un ensemble d'individus acceptant de payer, bien qu'ils n'aient pas de préférence pour la pêche au poisson sauvage. II s'agit de « non-usagers potentiels », leur $C A P$ peut s'interpréter comme étant l'expression monétaire de la valeur d'existence du poisson sauvage. Cette catégorie des pêcheurs a un certain nombre de caractéristiques qu'il convient de souligner. Leurs revenus tout d'abord sont plus élevés, on observe une sur-représentation des classes de revenu supérieures à $12500 \mathrm{~F}$. Par ailleurs ce sont des pêcheurs moins assidus et moins intéressés par la truite. La moyenne et la médiane de la distribution du CAP sont respectivement égales à $60 \mathrm{~F}$ et $20 \mathrm{~F}$, et sont donc inférieures à celles de l'ensemble de la population. La distribution de la valeur d'existence est concentrée entre $0 \mathrm{~F}$ et $50 \mathrm{~F}$.

\section{CONCLUSION SUR LA RENTABILITE SOCIALE}

La généralisation d'une gestion patrimoniale, centrée sur la restauration du milieu, est la finalité du programme. Ce choix de gestion, repose exclusivement sur la production spontanée du milieu en poissons sauvages. Le CSP apporte un soutien financier aux projets des associations de pêcheurs à raison d'environ 20 MF/an. De 1993 à 1999, les aides spécifiques du CSP aux travaux de réhabilitation ont été multipliées par 2,5 et ont ainsi accompagné les efforts des pêcheurs dans le montage des projets en faveur des milieux aquatiques (Figure 2). Enfin, les participations financières extérieures (Etat, collectivités locales, ...) à ces projets ont globalement été multipliées par quatre prouvant que les pêcheurs sont suivis dans leur démarche lorsqu'elle s'inscrit dans la politique nationale en faveur de l'environnement.

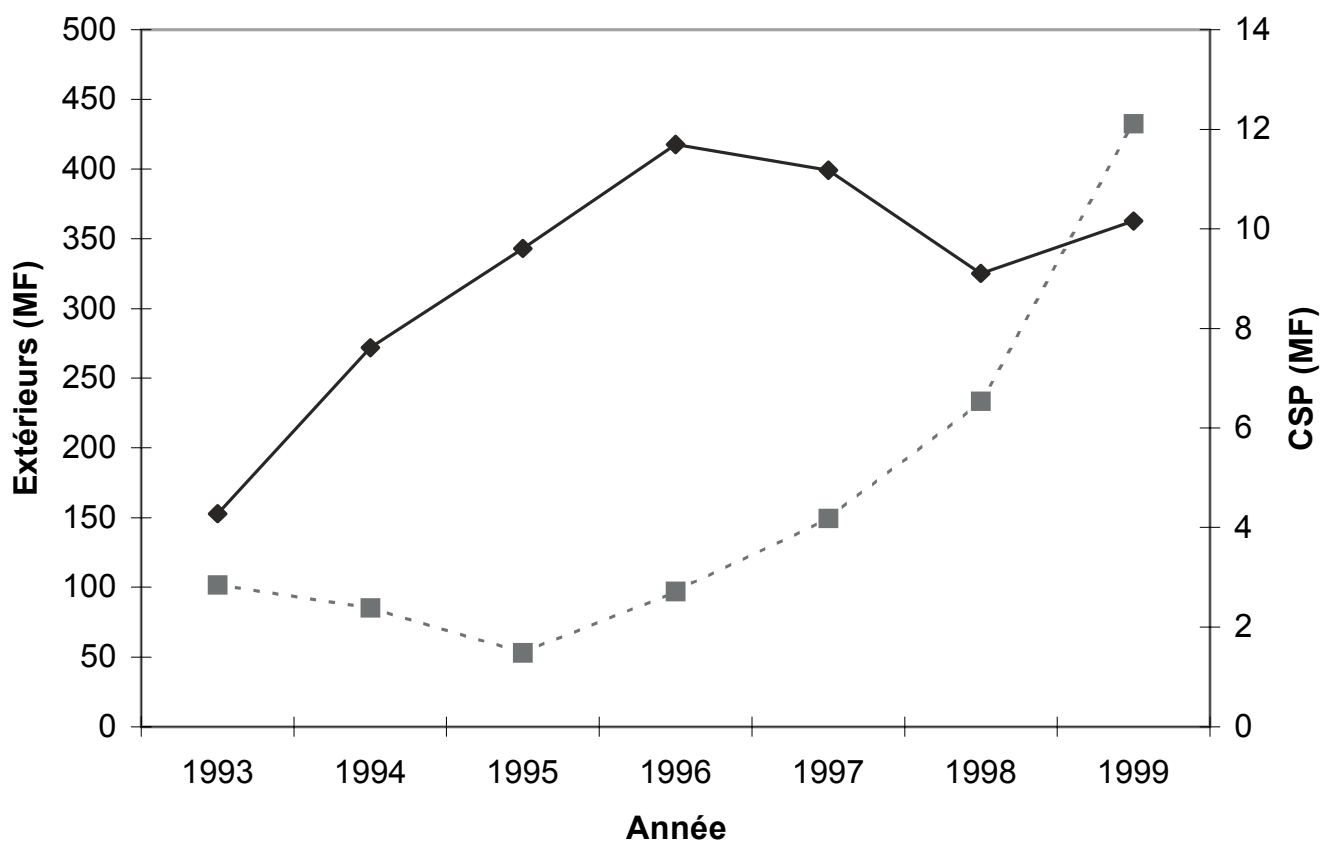

\section{Figure 2}

Evolution des financements apportés aux projets de réhabilitation de milieux. (contribution du CSP en traits plein et contributions extérieures en pointillés).

\section{Figure 2}

Evolution of the financial support to ecosystem restoration programme. (solid line represents the contribution of the CSP, dotted line represents outside contributions). 
Le coût des travaux à mettre en œuvre, rapporté au nombre de poissons sauvages adultes produits, se révèle très élevé dans la plupart des cas. Le coût d'une truite sauvage varie en effet suivant les contextes de 100 à $2000 \mathrm{~F}$, alors que celui d'un poisson de repeuplement est compris entre 30 et $470 \mathrm{~F}$ par poisson capturé (RICHARD, non daté). Cette comparaison partielle ne permet cependant pas de conclure que les travaux de réhabilitation ne sont pas socialement justifiés. La valeur du poisson sauvage ne se réduit pas à celle du poisson de remplacement, produit en pisciculture, mais intègre des composantes liés à la pratique de la pêche et au non-usage de la ressource dont bénéficie une population plus large que celle des pêcheurs. Celle-ci peut être sensible aux avantages globaux pour l'écosystème des actions de réhabilitation, le poisson sauvage étant un symbole de qualité des milieux aquatiques. A cet égard la lutte contre la pollution de l'eau des rivières et des lacs est la seconde préoccupation des français en matière d'environnement juste après la réduction de la pollution atmosphérique (IFEN, 1999). La sauvegarde des plantes et des animaux apparaît en cinquième position après les problèmes de déchets et la promotion de technologies respectueuses de l'environnement.

Le Tableau III fournit une évaluation de la valeur du poisson sauvage pour l'Indre et l'Hérault. II s'agit de bornes inférieures puisque l'on a utilisé les bornes inférieures des CAP estimé, respectivement $50 \mathrm{~F}$ pour les usagers potentiels ( $86 \%$ des pêcheurs) et $20 \mathrm{~F}$ pour les non-usagers potentiels. Ce choix est en particulier justifié par les biais d'autosélection, les pêcheurs les plus chevronnés s'étant davantage impliqués dans l'enquête sont susceptibles d'avoir un CAP plus élevé que ceux qui n'ont pas répondu.

\section{Tableau III}

Evaluation minimale par les pêcheurs de la valeur annuelle du poisson sauvage.

\section{Table III}

Anglers' minimum annually value of wild fish.

\begin{tabular}{|l|l|c|c|c|}
\hline \multirow{5}{*}{ Indre } & $\begin{array}{l}\text { Catégories } \\
\text { Categories }\end{array}$ & $\begin{array}{c}\text { Effectifs } \\
\text { Numbers }\end{array}$ & $\begin{array}{c}\text { CAP unitaire (F) } \\
\text { Unitary WTP (FF) }\end{array}$ & $\begin{array}{c}\text { CAP total (10 } \mathbf{F}^{3} \text { ) } \\
\text { Total WTP (FF 10 }\end{array}$ \\
\hline \multirow{5}{*}{ Hérault } & $\begin{array}{l}\text { Usagers } \\
\text { Users } \\
\text { Non-usagers } \\
\text { Non users }\end{array}$ & 2142 & 50 & 658 \\
\cline { 2 - 5 } & $\begin{array}{l}\text { Ensemble } \\
\text { All }\end{array}$ & 15300 & 20 & 43 \\
\hline & $\begin{array}{l}\text { Usagers } \\
\text { Users } \\
\text { Non-usagers } \\
\text { Non users }\end{array}$ & 3135 & 50 & 901 \\
\cline { 2 - 5 } & $\begin{array}{l}\text { Ensemble } \\
\text { All }\end{array}$ & 22390 & 20 & 63 \\
\hline
\end{tabular}

Note : $C A P$ désigne le consentement à payer.

Note: WTP is willingness to pay. 
Dans l'Indre et l'Hérault, le PDPG a été défini respectivement en mars 1998 et en avril 1999, et une première estimation des coûts d'investissement et de fonctionnement correspondants est disponible (Tableau IV). Le PDPG porte sur 46 contextes dont 15 en gestion patrimoniale dans l'Indre, et sur 47 contextes dont 18 en gestion patrimoniale dans l'Hérault. Le choix de la gestion patrimoniale est lié aux potentialités offertes par le milieu et à la volonté des pêcheurs de cesser les alevinages, aussi des proportions plus importantes de contexte en gestion patrimoniale sont attendues dans d'autres départements. Les bénéfices minimaux des pêcheurs sont deux fois plus élevés que les coûts annuels de fonctionnement dans l'Hérault et sont du même ordre dans l'Indre. Certes les bénéfices minimaux des pêcheurs ne permettent pas de couvrir à la fois les coûts annuels de fonctionnement et l'amortissement si les travaux sont amortis sur cinq ans. Toutefois, au choix de valeurs minimales pour le CAP, s'ajoutent deux autres sources de sous-estimation : non-prise en compte de l'effet revenu et calcul limité aux seuls pêcheurs. Comme on l'a montré, le $C A P$ est une fonction croissante du revenu, la croissance du niveau de vie entraînera donc, toutes choses égales par ailleurs, celle du CAP dans l'avenir. C'est un phénomène classique avec les biens supérieurs et en particulier les biens environnementaux. Par ailleurs, certains non-pêcheurs accordent une valeur aux habitats naturels et au poisson sauvage qui n'est pas intégrée dans le calcul. En l'absence d'élément sur sa valeur moyenne et sur la population concernée, il serait erroné en l'état des connaissances de tirer une conclusion sans nuance sur la rentabilité sociale de ces plans.

\section{Tableau IV}

Coût du Plan Départemental pour la Protection du milieu aquatique et la Gestion des ressources piscicoles (PDPG) de l'Indre et de l'Hérault.

\section{Table IV}

Management scheme (PDPG) costs in Indre and Hérault.

\begin{tabular}{|c|c|c|}
\hline Département & 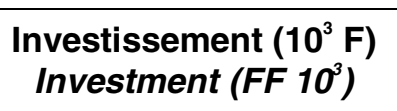 & $\begin{array}{l}\text { Fonctionnement (10 } 10^{3} \text { F/an) } \\
\text { Operating costs (FF 10 } 0^{3} / \text { year) }\end{array}$ \\
\hline Indre & 4000 & 700 \\
\hline Hérault & 5000 & 500 \\
\hline
\end{tabular}

\section{REMERCIEMENTS}

Les auteurs remercient pour leurs critiques et leurs conseils deux lecteurs anonymes de la revue.

Recherche ayant bénéficié d'une aide du Conseil Supérieur de la Pêche (convention d'étude C 30111). 


\section{BIBLIOGRAPHIE}

ANONYME, 1996. Résultats de synthèse et premières indications stratégiques. In : " Enquête qualitative auprès des clientèles du tourisme halieutique », Cabinet d'audit stratégique CRISTAL, Paris, France, 69-93.

ARMAND C., BONNIEUX, F., 1999. Valeur du poisson sauvage et rentabilité sociale des plans de gestion piscicole. Communication présentée aux Journées « Economie de l'Environnement » (Strasbourg 2-3 décembre 1999), 23 p.

ARROW K., SOLOW R., PORTNEY P.R., LEAMER E.E., RADNER R., SCHUMAN H., 1993. Report of the National Oceanic and Atmospheric Administration panel on contingent valuation. Federal Register, 58, January 15, 4601-4614.

BONNIEUX F., DESAIGUES B., 1998. Economie de l'environnement. Dalloz, Paris, 328 p.

BATEMAN I.J., WILLIS K.G. (Eds.), 1999. Valuing environmental preferences. Oxford University Press, $645 \mathrm{p}$.

FREEMAN A.M. III, 1993. The measurement of environmental and resource value, theory and methods. Resources for the Future. Washington D.C., 516 p.

GAMOND J., 1991. Synthèse des résultats de l'enquête nationale réalisée sur la pêche en eau douce : rapport final. Institut de sondage Lavialle, Issy-les-Moulineaux, France, $242 \mathrm{p}$.

HOLL M., AUXIETRE J.P., non daté. Gestion piscicole et plans de gestion : conception et pratique. Collection « Mise au point », Conseil Supérieur de la Pêche, Paris, France, $240 \mathrm{p}$.

IFEN, 1999. L'opinion publique sur l'environnement et l'aménagement du territoire en 1998. Orléans, Institut Français de l'Environnement, Observatoire des Pratiques et des Représentations Sociales, Collection « Etudes et Travaux 》 $\mathrm{n}^{\circ} 22,111 \mathrm{p}$.

JANTZEN J.M., 1998. A national survey on freshwater fishing in France. In : Recreational Fisheries: social, economic and management aspects, HICKLEY P. and TOMPKINS H. (Eds.). Oxford, U.K., Fishing News Books, 5-9.

POLLOCK K.H., JONES C.M., BROWN T.L., 1994. Angler survey methods and their applications in fisheries management. American Fisheries Society, Bethesda, Maryland, $369 \mathrm{p}$.

RICHARD A., non daté. Gestion piscicole : interventions sur les populations de poissons, repeuplement des cours d'eau salmonicoles. Collection « Mise au point ", Conseil Supérieur de la Pêche, Paris, France, $256 \mathrm{p}$.

SOUCHON Y., TROCHERIE F., 1990. Technical aspects of French legislation dealing with the freshwater fishery (June 1984): «Fisheries orientation schemes » and " Fishery resources management plans ». In: Management of freshwater fisheries. (Proceedings of the EIFAC symposium, Göteborg, Sweden). VAN DENSEN W.L.T., STEINMETZ B. and HUGHES R.H. (Eds.). Wageningen. 190-214.

WILLIS K.G., GARROD G.D., 1999. Angling and recreation values of low-flow alleviation in rivers. Journal of Environmental Management, 57, 71-83. 